\title{
Care of Maintenance Hemodialysis Patients through Intelligent Algorithm-Based Low-Dose Digital Subtraction Angiography
}

\author{
Jianhua Hong (iD \\ Department of Gastroenterology, Tonglu First People's Hospital, Tonglu, Hangzhou 311500, Zhejiang, China \\ Correspondence should be addressed to Jianhua Hong; 13261065@bjtu.edu.cn
}

Received 18 September 2021; Accepted 29 October 2021; Published 30 November 2021

Academic Editor: M Pallikonda Rajasekaran

Copyright (c) 2021 Jianhua Hong. This is an open access article distributed under the Creative Commons Attribution License, which permits unrestricted use, distribution, and reproduction in any medium, provided the original work is properly cited.

\begin{abstract}
Objective. The study aimed to explore the application value of artificial intelligence (AI)-based low-dose digital subtraction angiography (DSA) in the care of maintenance hemodialysis (MHD) patients. Methods. The characteristics of DSA imaging were analyzed, and the refinement efficiency of the AI algorithm was discussed, expected to assist clinicians in the care and treatment of patients. $100 \mathrm{MHD}$ patients who were in the hospital were selected as the research subjects. They were randomly divided into the conventional DSA group (conventional group) and the AI algorithm-based DSA group (AI-based DSA group). The conventional group used conventional DSA images to guide the care of HM patients, and the AI-based DSA group used the AI algorithm to optimize DSA images. Results. It was found that the AI-based DSA group was better than the conventional DSA group in terms of image sharpness and shaded areas, and the image mean square error (MSE) loss value was smaller $(P<0.05)$. The patients were followed up for 3 months. In the AI-based DAS group, the blood flow of the drainage vein (DV), the blood flow of the proximal vein (PA), and the blood flow of the brachial artery (BA) were greater than those of the conventional group $(P<0.05)$. During the 3-month follow-up period, in the conventional group, thrombosis occurred in 4 patients, low-flow AVF occurred in 5 patients, high-flow AVF occurred in 3 patients, and heart failure occurred in 5 patients. In the AI-based DSA group, thrombosis occurred in 2 patients, low-flow AVF occurred in 2 cases, high-flow AVF occurred in 1 case, and heart failure occurred in 3 cases. There were no other cardiac complications in both groups. Conclusion. DSA images optimized by the AI algorithm are suitable for clinical diagnosis and have practical application value.
\end{abstract}

\section{Introduction}

Chronic kidney disease (CKD) is a global concern [1]. Statistics reveal that the incidence of CKD in China is as high as $10.8 \%$, and $\mathrm{CKD}$ is often diagnosed in the late stage of kidney disease. Hemodialysis (HD) is the main treatment method for CKD patients [2]. Blood vessels are considered to be the lifeline of HD patients, and the dialysis rate of longterm dialysis patients for HD within 1 year is $60 \%$, and the medical cost is 2 to 3 times that of the ordinary patients [3]. Therefore, effective countermeasures are required to reduce unnecessary medical expenses.

Digital subtraction angiography (DSA) is a medical imaging system based on X-rays used in clinical angiography [4]. DSA is necessary for the treatment of vascular diseases. It is not only widely used in the diagnosis of general vascular diseases but also used in clinical interventional therapy [5]. DSA images have high resolution and a wide range of gray levels, which can accurately present blood vessel. With the rapid development of medical image processing technologies, the DSA equipment has also been improved. Together, there are new requirements for the diagnosis of vascular diseases. DSA has problems such as the low quality of blood vessel subtraction images and high radiation dose, and the there is a lot of noise in the final DSA image. Except for the poor compliance of the patient and high-dose radiation, there exist artifacts [6]. When X-rays pass through the body, they are absorbed by the body tissue. Nevertheless, longterm exposure to X-ray will cause irreversible damage to human cells and DNA. In severe cases, it sometimes causes leukemia or cancer. Hence, reducing the radiation amount during DSA is a hot spot [7]. 
DSA image optimization is similar to traditional medical image enhancement, which mainly includes image enhancement and noise removal [8]. Image enhancement can filter background information according to the logarithmic correction method of X-ray attenuation rule [9]. The frame overlapping method can reduce random noise. In order to enhance the contrast of the background and blood vessels of DSA images, adaptive histograms are used to increase the gray level of local areas [10]. In areas with small gray-scale fluctuations, the contrast is poor, while in areas with large gray-scale fluctuations, there is too much artifact information. He et al. proposed that scale morphological enhancement can maintain most of the vascular structure, but it loses some vascular details and has low anti-noise strength [11]. Although postprocessing of DSA images heightens the doctor's diagnostic efficiency, the patient still suffers from high radiation doses due to the inability to clear artifacts [12].

The noise elimination of deep convolutional neural network (CNN) is realized by the network loss function. The entire network is a process of learning noise residuals, and the output results are significantly better than traditional algorithms [13, 14]. Deep learning-based image processing requires multiple output tags corresponding to the input training data. Consequently, CNN can be applied to eliminate artifacts in DSA images, so as to reduce the radiation doses. Deep learning-based feature extraction extracts DSA vascular subtraction from a single frame to eliminate the dependence on the background frame and reduce the exposure time and radiation dose [15].

In this study, the back-projection filtering algorithm was used and optimized. Image refinement based on intelligent algorithms can improve nursing efficiency and reduce radiation dose and side effects. The peak signal-to-noise ratio (PSNR) was used to detect the noise reduction efficiency and study the vascular imaging characteristics in vascular dialysis.

\section{Materials and Methods}

2.1. Research Subjects. In this study, 100 patients with MHD in the hospital from April 2019 to April 2021 were selected as research subjects. They were randomly divided into the conventional DSA group (conventional group) and artificial intelligence-based DSA group (AI-based DSA group). The two groups of patients exercised for 20 minutes every day on non-dialysis days. Vascular conditions were detected with Doppler ultrasound. The curative effect indexed included the blood flow of the drainage vein (DV), proximal artery (PA) blood flow, carotid artery (CA) blood flow, DV diameter, and incidence of adverse events.

The patients were selected as per the following inclusion criteria: (I) over 18 years old; (II) receiving MHD treatment for 3 months; and (III) good condition on dialysis.

Exclusion criteria were as follows: (I) the vascular retention tube was not removed; (II) arterial diameter $>2 \mathrm{~cm}$ or exposure to the risk of rupture; (III) participating in other clinical trials at the same time; and (IV) with other blood diseases.
Withdrawal criteria were as follows: (I) requiring other surgeries and (II) accompanied by other diseases.

In this study, a total of $100 \mathrm{MHD}$ patients were identified. This study has been approved by the Medical Ethics Committee of the hospital, and the family members of the patients included in the study had signed an informed consent form.

2.2. Dialysis Methods. The HD device manufactured by Belem, Germany, was used. The dialysate was bicarbonate, and a polysulfone membrane dialysis device was used. During $\mathrm{HD}$, to improve the anti-coagulation effect and reduce the side effects of bleeding, low-molecular-weight serine calcium was used as the main anti-coagulant for dialysis treatment.

2.3. DSA Examination. During DSA, epidural anesthesia was used and the patient was in the supine position. Affected by the femoral artery, the lesions on both sides or one side were examined for anterograde or retrograde using the Seldinger technique. The human catheter was placed on the patient, and $2-13 \mathrm{~mL}$ of contrast agent was injected through a double-barrel high-pressure cylinder at a rate of $4-7 \mathrm{~mL}$ per second. Then, the patient was scanned from the horizontal position of the renal artery to the sole of the foot.

2.4. DSA Imaging. The low-dose DSA algorithm can reduce the radiation dose and obtain high-quality DSA vascular subtraction images without motion artifacts. The existing research on low-dose DSA algorithm is mainly to reduce radiation dose and remove artifacts. However, there are limitations. CNN has powerful non-linear mapping ability and can automatically extract global semantic features and local edge features of images. In recent years, deep learning has developed rapidly in the field of medical image processing. Based on deep learning, this study aimed to reduce the radiation dose of DSA vascular subtraction, remove the motion artifacts, and reduce the radiation dose received by patients while providing powerful assistance to interventional diagnosis and treatment. DSA imaging involves the injection of a contrast agent into the patient's blood vessel and subtraction of two images. Essentially, DSA refers to continuous X-ray images. Due to the different attenuation of human tissue to X-rays, corresponding gray-scale information is obtained for each frame of the image. The light intensity attenuates with the density and thickness of human tissue, which conforms to the Lambert-Beer law.

$$
A=\lg \left(\frac{1}{T}\right)=K b c
$$

where $A$ is the absorbance; $T$ is the transmittance, namely, the ratio of the intensity of outgoing light $(I)$ to the intensity of incident light $\left(I_{0}\right) ; K$ is the molar absorption coefficient, and it is related to the absorbing material and the wavelength $\lambda$ of the incident light; $c$ is the concentration of the lightabsorbing substance, and the unit is $\mathrm{mol} / \mathrm{L}$; and $b$ is the thickness of the absorbing layer. The mask image taken 
before the injection of the contrast agent can be expressed as follows:

$$
\omega_{l}=\alpha \omega_{0} e^{-\left(\mu_{0} \chi_{0}+\mu_{1} \chi_{1}\right)},
$$

where $\omega$ is the attenuation coefficient of human tissue and $a$ is the signal amplification factor during X-ray radiation. After the contrast agent is injected, the contrast frame (live film) is expressed as follows:

$$
\omega_{1}=\alpha \omega_{0} e^{-\left(\mu_{0} \chi_{0}+\mu_{1} \chi_{1}\right)},
$$

where $\omega_{1}$ is the attenuation coefficient of the contrast agent in the blood vessel. For X-ray images, the gray level is 4096 , black is 0 , and white is 4096 . One image is subtracted from the other to obtain the retained blood vessel information. The whole process is expressed as follows:

$$
I_{d s a}=\operatorname{In}\left(\omega_{m}\right)-\operatorname{In}\left(\omega_{l}\right)=\left(-\mu_{0} \chi_{0}\right)-\left(-\left(\mu_{0} \chi_{0}+\mu_{1} \chi_{1}\right)\right)=\mu_{1} \chi_{1} \text {. }
$$

Figure 1 shows the flowchart.

The vascular subtraction after logarithmic operation is not affected by bones and soft tissue. Figure 2 shows the entire DSA imaging process.

2.5. Medical Image Denoising. The DSA images have many disadvantages. Especially in X-ray photography, information collection, transmission components, and information output are affected by different magnitudes of quantum noise. Artificial intelligence-based DSA imaging requires vascular subtraction labels with almost no noise and artifacts [16]. Reducing the influence of distance information on edge information can save high-frequency edge information. The blood vessel subtraction image including noise information is denoted as $f=f$, and the coordinates of any point $P$ are $(x, y) . K$ is the neighborhood range of the bilateral filter width, expressed as follows:

$$
\Omega_{p}(K)=\{P+(i, j):-K \leq i, j \leq N\} .
$$

The pixel point after bilateral filtering operation is $f(p)=\sum_{y=\Omega} E(x, y) u(y) / \sum_{y=\Omega} E(x, y)$, where $\sum_{y=\Omega} E(x, y)$ is the weight function of bilateral filtering, defined as follows.

where $E(x, y)$ and $E_{s}(x, y)$ are the spatial proximity factor and the brightness similarity factor, respectively, and $\sigma_{1}^{2}$ and $\sigma_{K}^{2}$ represent the Gaussian kernel variance that controls the similarity factor. Histogram equalization maps the gray level of pixels, stretches the gray value of the entire image range to an average shadow, and improves the contrast and saturation of the image. The gray level transformation function $T(r)$ ensures that the order of the pixel gray levels remains unchanged after the transformation and maintains the consistency of the gray dynamic range before and after the transformation. $r$ is the normalized gray level, and $n$ is the total number of pixels. The histogram equalization function of the image is as follows:

$$
\begin{aligned}
& E(x, y)-E_{s}(x, y) E_{R}(x, y) ; \quad E_{s}(x, y) \\
& \quad=e^{(x-y)^{2} / 2 \sigma_{1}^{2}} ; E_{R}(x, y)=e^{\left|f(x)-f(y)^{2}\right| / 2 \sigma_{k}^{2}},
\end{aligned}
$$

where $k$ is the gray level of the image. The gray level transformation function Tmaps the $r$-level gray pixels in the image to $H$-level gray pixels.

2.6. Artificial Intelligence-Based Angiography. Montoya et al. proposed deep learning-based angiography (DLA) and gave the corresponding vascular subtraction image. The DLA is a classification model of pixel blocks. The voxels of angiographic bone are divided into 3 categories: blood vessels, bones, and soft tissue. The clinical data are divided by artificial thresholds, and small areas of shadows are considered residual artifacts. The blood vessel label is obtained by filtering motion artifacts and background noise. Numerical segmentation and connectivity analysis are performed in the background frame. Soft tissue labeling is performed by angiographic segmentation. The CNN-based angiography can effectively solve the artifacts without relying on the background frame. It eliminates the necessity of background frame collection and reduces the radiation dose. The process of obtaining classification labels is shown in Figure 3.

2.7. DSA Image Quality Evaluation. Image quality evaluation (IQE) was used to scientifically evaluate the quality of DSA images. Image quality evaluation methods include subjective evaluation and objective evaluation. The subjective evaluation requires multiple experiments, and the imaging effects are determined based on the subjective factors of experts. Objective evaluation quantifies the quality of vascular subtraction based on mathematical models and scientifically determines the quality of overall and partial medical images. For example, mean square error (MSE) is used to measure the difference between estimators. The objective evaluation method is based on the absolute error between pixels. Due to the specificity of DSA images, blood vessels should be clearly displayed. MSE is expressed as follows:

$$
\operatorname{PSNR}=10 \log _{10}\left(\frac{255^{2} \times M \times N}{\sum_{w=1}^{M} \sum_{e=1}^{N} u(w, e)-f(w, e)^{2}}\right),
$$

where $M \times N$ represents the size of CT and $u$ and $f$ are the original image and the noise-removed image, respectively. A higher PSNR value indicates a smaller MAE and better image noise reduction effects.

Structural similarity difference (SSIM) is expressed as follows:

$$
\begin{aligned}
\operatorname{MSSIM}(u, f) & =\frac{1}{N} \sum_{w=1}^{N} \operatorname{SSim}\left(x_{w}, y_{w}\right), \\
\operatorname{SSIM}(x, y) & =\frac{\left(2 \alpha_{x} \alpha_{y}+c_{1}\right)\left(2 \sigma_{x y}+c_{2}\right)}{\left(\alpha_{x} \alpha_{y}+c_{1}\right)\left(\sigma_{x}{ }^{2}+\sigma_{y}{ }^{2}+c_{2}\right)},
\end{aligned}
$$

where $u$ and $f$ represent the original image and the noiseremoved image, $X$ and $Y$ represent the original image and the noise, $N$ represents the average value of the gray value of the pixel, that is, the dispersion of the gray value of the pixel of the image, and $C 1$ and $C 2$, which are the covariances of 


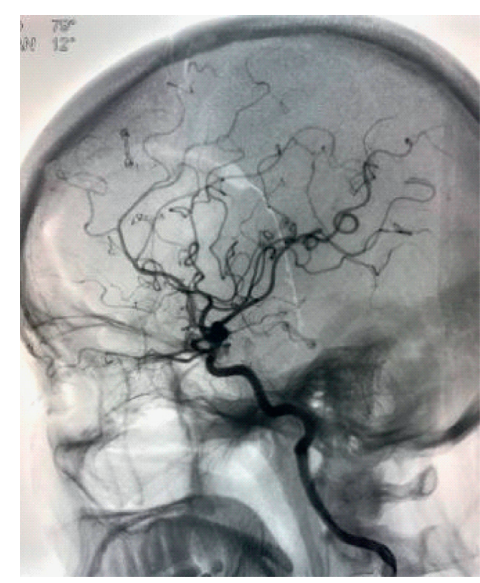

Step 1

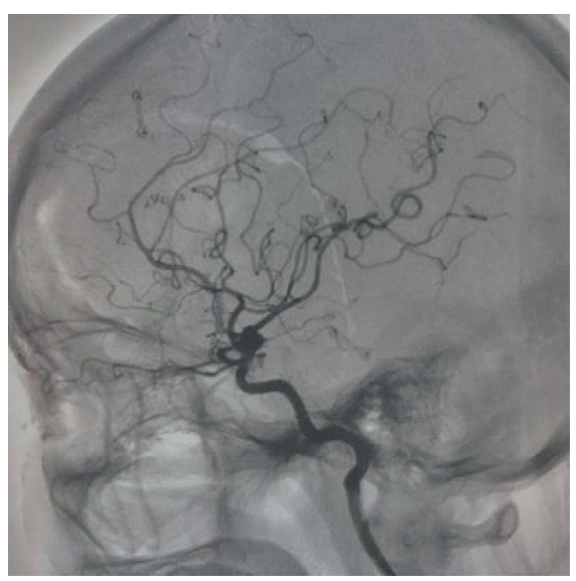

Step 2

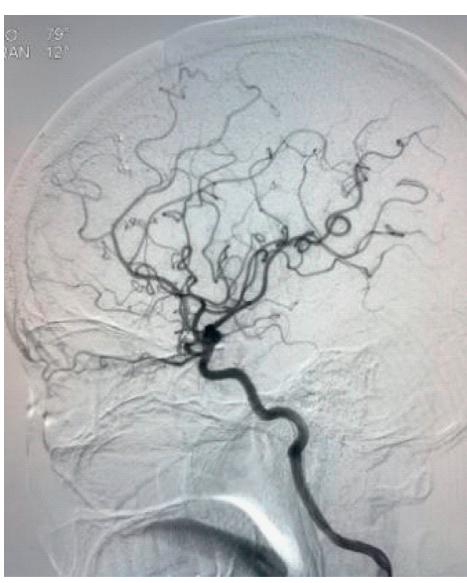

Step 3

FIGURE 1: DSA imaging principle. (a) Step 1: left internal carotid angiography, lateral mask image. (b) Step 2: left internal carotid angiography, lateral live film. (c) Step 3: subtraction of live film and mask image, left internal carotid artery angiography.

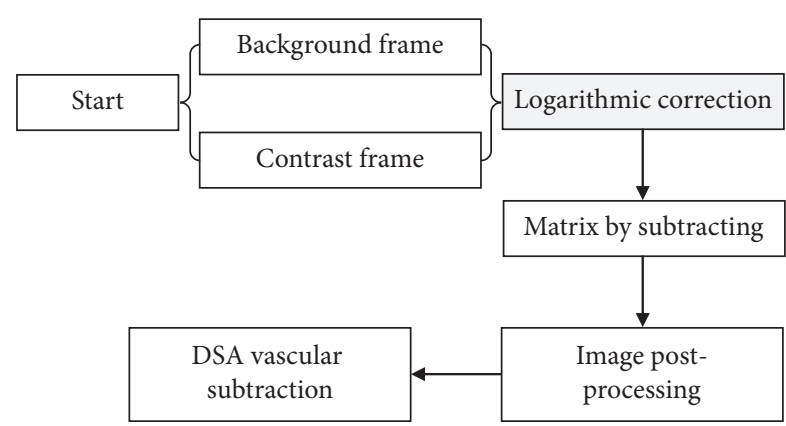

Figure 2: DSA imaging process.

image blocks $x$ and $y$, are small constants to guarantee that the denominator is not zero. A larger SSIM value indicates that the noise-removed image and the original image are more similar.

2.8. Network Training and Testing. The DSA artifact removal network has an end-to-end structure, and the parameters of the training process are set as follows. The learning rate is 0.0001 , the amount of input data is 8 , and the number of network iterations is 100 . The complexity of the online extended dataset and the network model determines the training time of the model, which is about 12 hours.

2.9. Statistics. The data were processed by SPSS21.0. The result of DSA was the gold standard, that is, the data were consistent with the normal distribution, so the mean\pm standard deviation $(x(-) \pm s)$ was used. $P<0.05$ was the threshold for significance.

\section{Results}

3.1. Experiment Results of Network Model. Evaluation of the image processed by the AI algorithm included objective quantitative evaluation and subjective qualitative analysis. The DSA image with artifacts was used as the network input data. An objective quality evaluation was carried out on the non-artifact test set. The subjective evaluation compared the images optimized by the conventional method and AI algorithm (Figures 4-6).

The subjective evaluation results showed that the motion artifacts were eliminated, and the image had high contrast, with blood vessel details well preserved. AI algorithm can remove artifacts and save blood vessel images. The AI algorithm based on deep learning was efficient and reduced the radiation dose.

In order to objectively evaluate the results of different network models, DSA image datasets of the brain and feet were tested. Figure 7 shows the average PSNR and SSIM of the lower limbs and cerebral vessels according to different methods. The results showed that DSA images optimized by the AI algorithm achieved good results in terms of PSNR and SSIM.

3.2. MSE Loss Values. This section compared the scatter points of the MSE loss value of the conventional method and the AI algorithm. The red dots represented the conventional method, and the black dots represented the AI algorithm. It was noted from Figure 8 that in the initial stage of AI training, it converged steadily and quickly, and the training loss value decreased and converged equally. This suggested that AI algorithm enabled a larger receptive field, and the dilated convolution also prevented information loss caused by increased pooling.

3.3. DV Blood Flow. The changes of DV blood flow within 3 months of follow-up were recorded, as shown in Figure 9. The interaction effect of DV blood flow was statistically different $(P<0.001)$, indicating that two DSA image processing methods had different effects on DV blood flow.

3.4. PA Blood Flow between the Two Groups. It was found that the difference between different time points was statistically significant $(P<0.001)$, indicating that the patient's PA blood flow changed with time. The PA blood flow at 1,2, and 


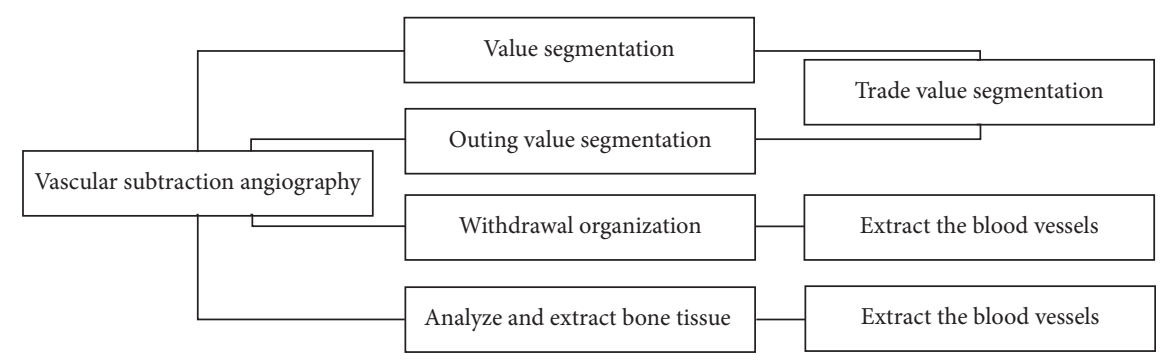

Figure 3: Label data acquisition process.

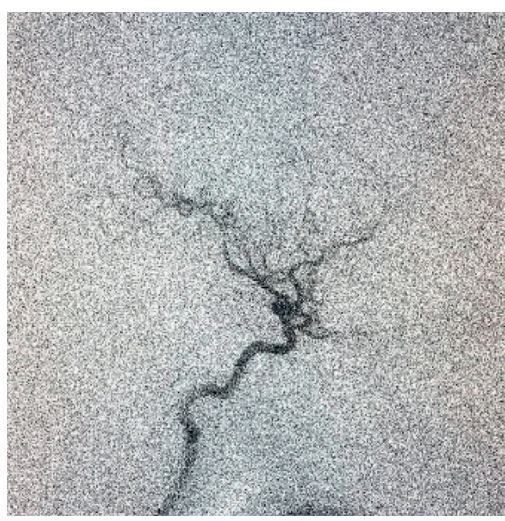

(a)

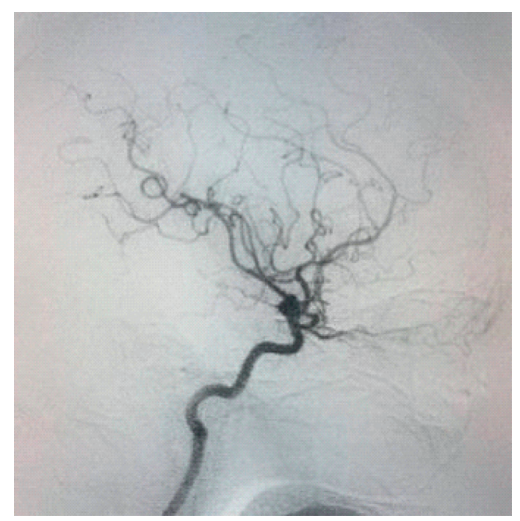

(b)

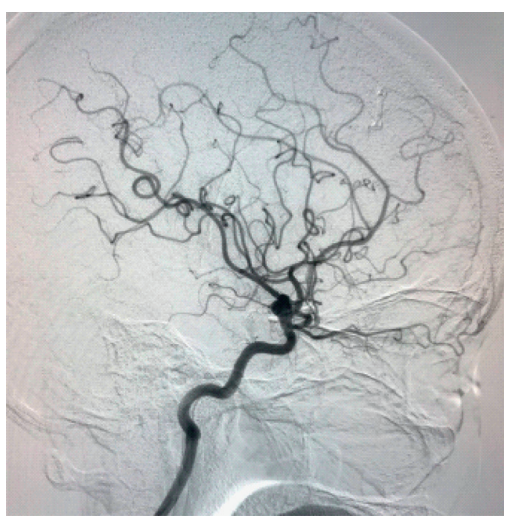

(c)

Figure 4: Noise reduction effects by the conventional method and AI algorithm. (a) Original image of blood vessel DSA image. (b) Image optimized by the conventional method. (c) Image optimized by the AI algorithm.

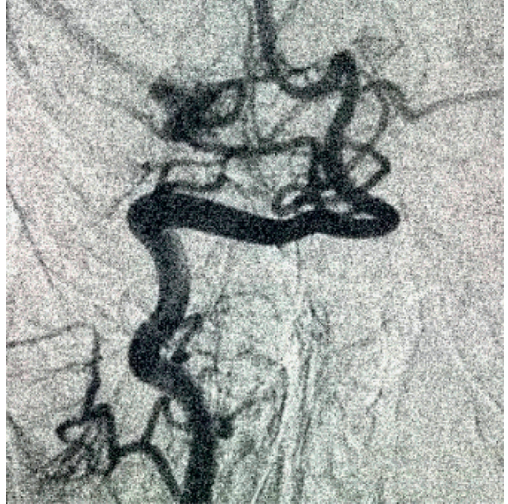

(a)

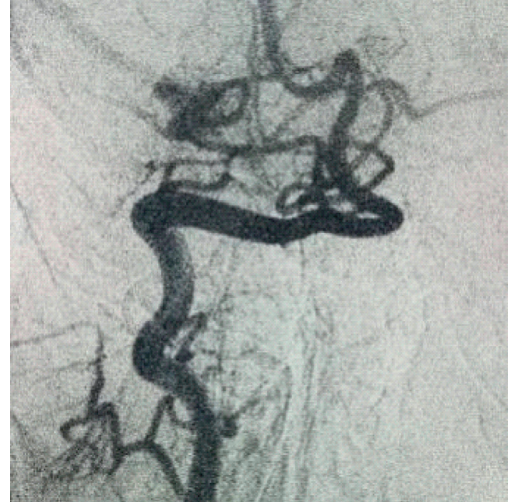

(b)

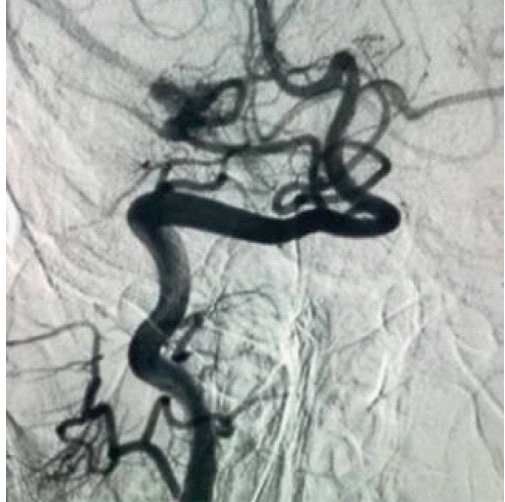

(c)

FIGURE 5: Image refinement effects by the conventional method and AI algorithm. (a) Original image of blood vessel DSA image. (b) Image optimized by the conventional method. (c) Image optimized by the AI algorithm.

3 months of follow-up was statistically different from that before the intervention $(P<0.05)$, as shown in Figure 10 .

3.5. BA Blood Flow between the Two Groups. It was found that the time had no statistically significant effect on BA blood flow $(P=0.176)$, indicating that there was no interaction between time and grouping; BA blood flow at 3 months after the intervention was statistically different from that at 2 months after the intervention $(P=0.013)$, as shown in Figure 11.

3.6. DV Diameters. It was found that the DV diameter at 2 months after the intervention was statistically different from that before the intervention $(P<0.05)$, and DV diameter at 3 months after the intervention was statistically different from that at 1 month after the intervention $(P<0.001)$; the difference was 0.45, as shown in Figure 12. 


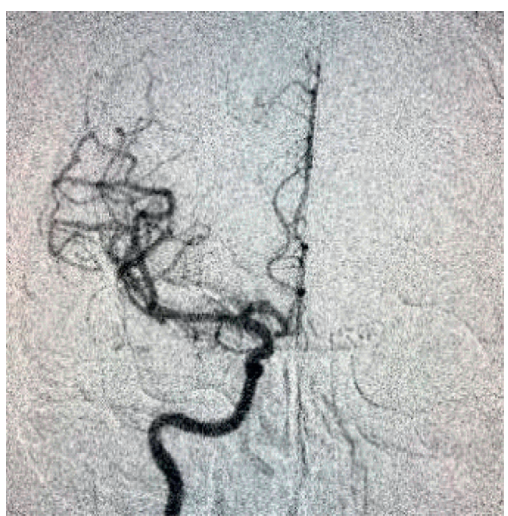

(a)

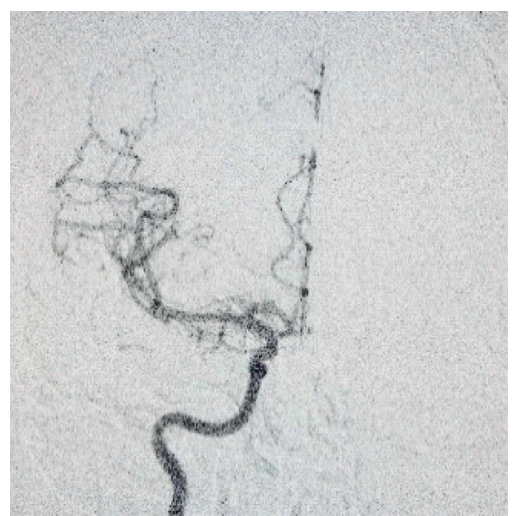

(b)

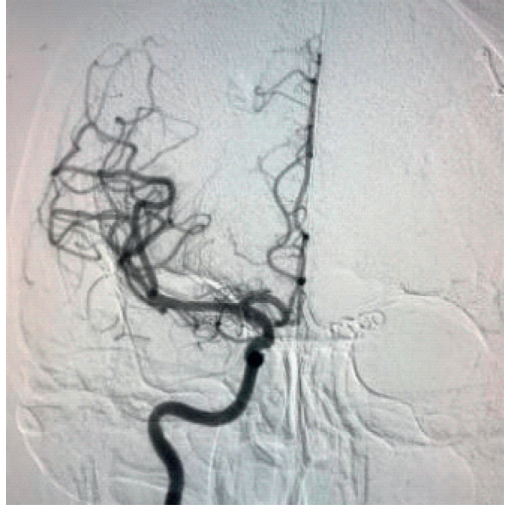

(c)

Figure 6: Anti-artifact effects by the conventional method and AI algorithm: (a) original image of blood vessel DSA image; (b) DSA image of blood vessel with conventional method. (c) Artifact-removed DSA image of blood vessel with AI algorithm.

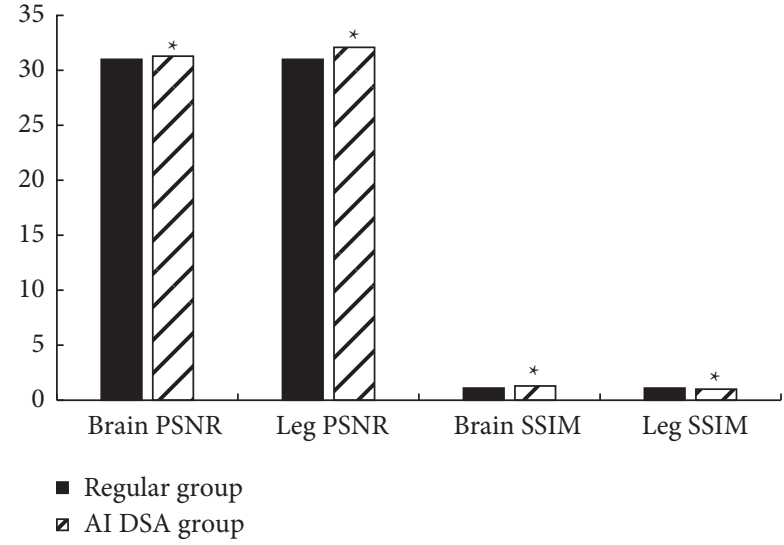

FIGURE 7: Average PSNR and SSIM of lower limbs and cerebral vessels $\left({ }^{*}\right.$ indicated that there were statistical differences between groups).

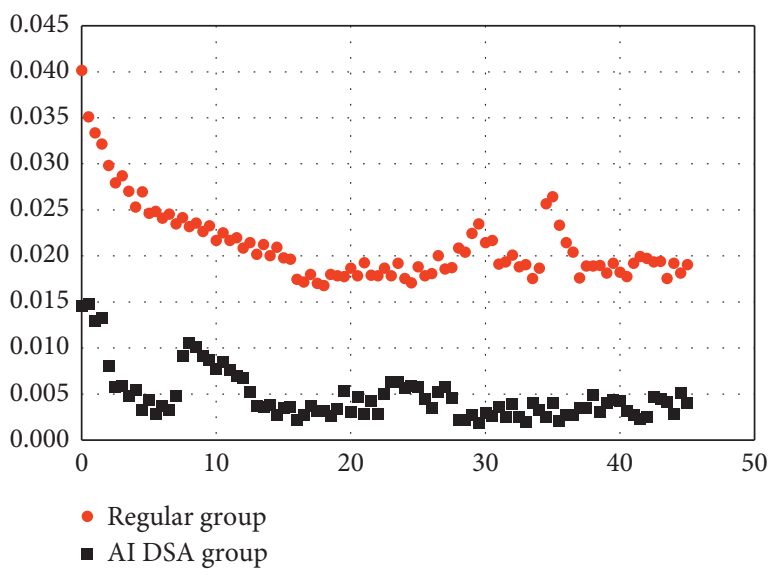

FIGURE 8: Scatter plots of MSE loss values of different network structures.

3.7. Adverse Reactions between the Two Groups. During the 3-month follow-up period, in the conventional group, thrombosis occurred in 4 patients, low-flow AVF occurred

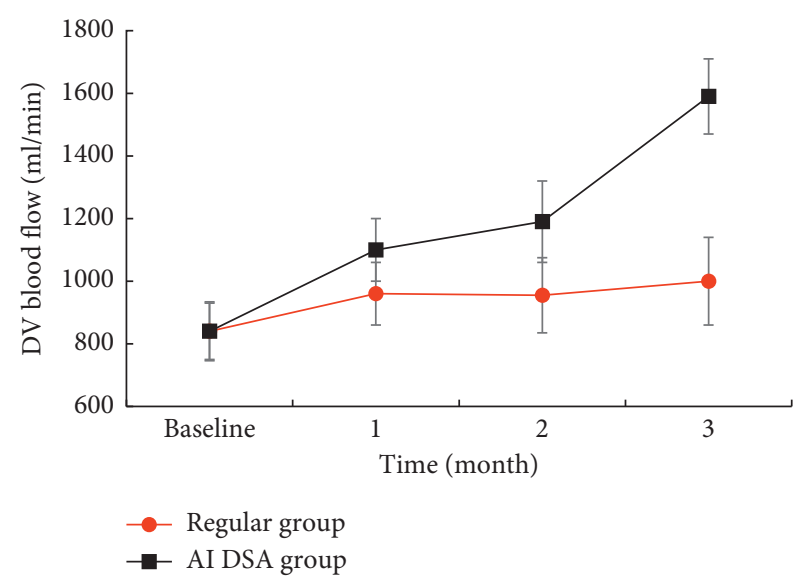

Figure 9: The DV blood flow of the two groups within 3 months of follow-up.

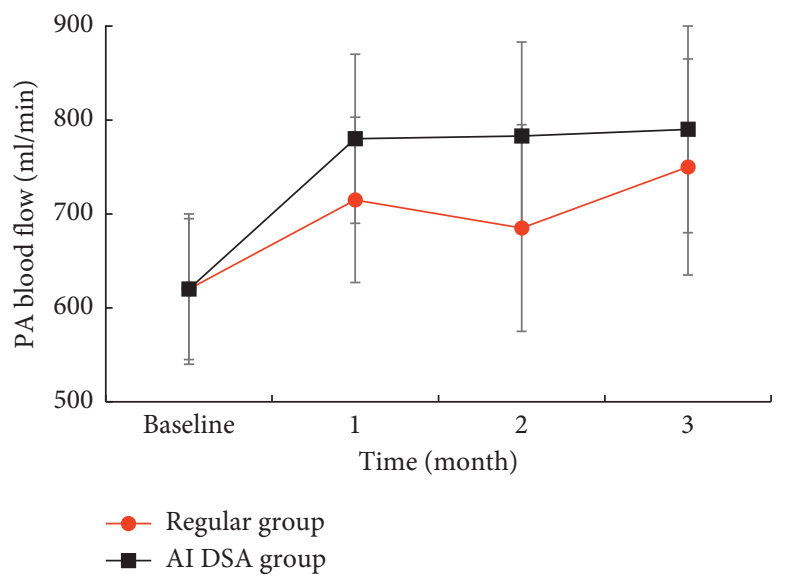

FIgURE 10: PA blood flow in the two groups within 3 months of follow-up.

in 5 patients, high-flow AVF occurred in 3 patients, and heart failure occurred in 5 patients. In the AI-based DSA group, thrombosis occurred in 2 patients, low-flow AVF 


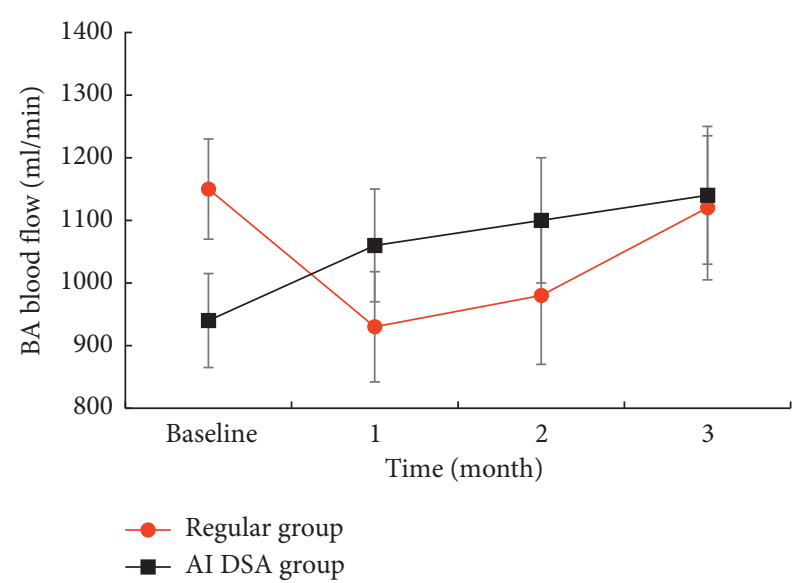

Figure 11: BA blood flow of the two groups within 3 months of follow-up.

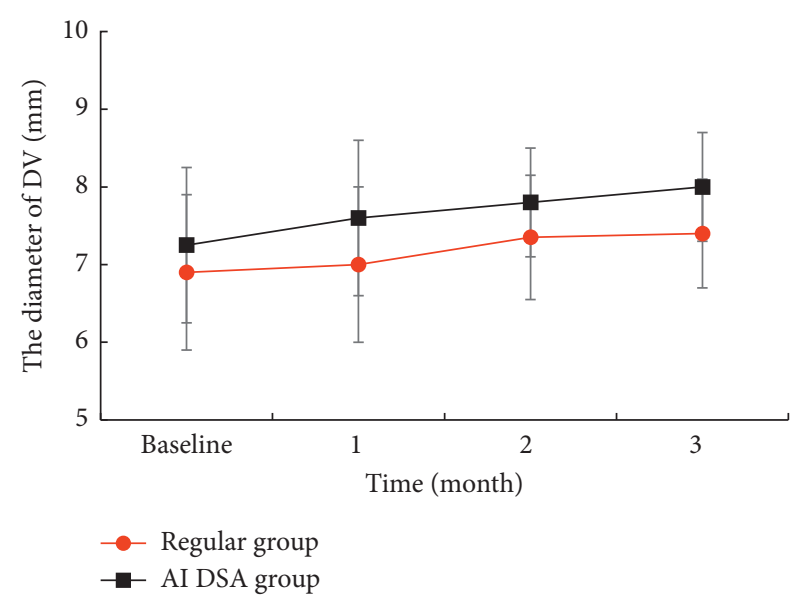

Figure 12: DV diameters of the two groups within 3 months of follow-up.

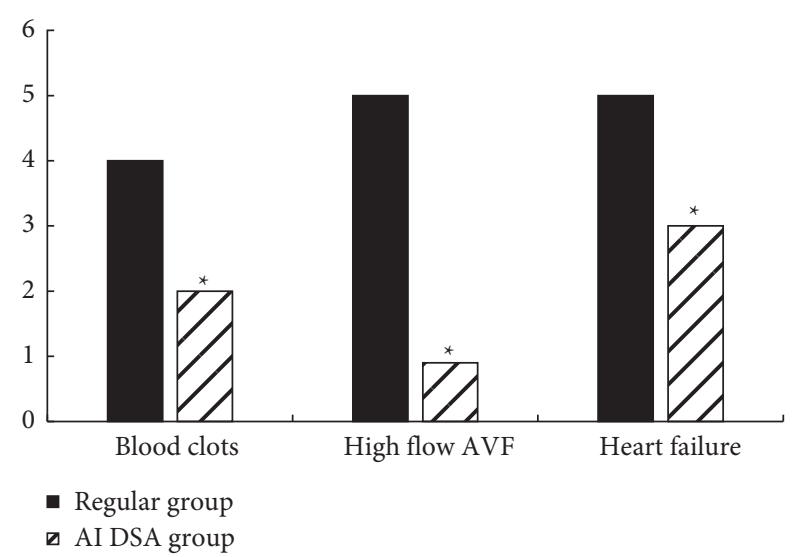

Figure 13: Adverse events between the two groups within 3 months of follow-up.

occurred in 2 cases, high-flow AVF occurred in 1 case, and heart failure occurred in 3 cases, as shown in Figure 13. There were no other cardiac complications in both groups.

\section{Discussion}

With the aging of the population, the incidence of CKD has shown a rapid increase [17]. End-stage kidney disease requires kidney replacement therapy, mainly $\mathrm{HD}$, peritoneal dialysis, and kidney transplantation. HD is an effective kidney replacement therapy. It executes the renal excretion function to eliminate blood metabolic wastes and electrolyte barriers. To maintain acid-base balance, different patients need distinct treatment plans. DSA can well observe the physiological conditions of the patient's blood vessels [18]. There was no statistically significant difference between the two groups at the beginning, while in January, DV blood flow was $971 \mathrm{~mL} / \mathrm{min}$ in the conventional group and $1079 \mathrm{~mL} / \mathrm{min}$ in the AI-based DSA group; in February, the DV blood flow in the conventional group was $977 \mathrm{~mL} / \mathrm{min}$, and that of AI-based DSA group was $1196 \mathrm{~mL} / \mathrm{min}$; in March, DV blood flow was $1001 \mathrm{~mL} / \mathrm{min}$ in the conventional group and $1592 \mathrm{~mL} / \mathrm{min}$ in the AI-based DSA group. There was significant difference between the two groups. There was no statistically significant difference between the two groups at the beginning, while in January, the PA blood flow was $713 \mathrm{~mL} / \mathrm{min}$ in the conventional group and $781 \mathrm{~mL} / \mathrm{min}$ in the AI-based DSA group; in February, PA blood flow was $689 \mathrm{~mL} / \mathrm{min}$ in the conventional group and $792 \mathrm{~mL} / \mathrm{min}$ in the AI-based DSA group; in March, PA blood flow was $684 \mathrm{~mL} / \mathrm{min}$ in the conventional group and $799 \mathrm{~mL} / \mathrm{min}$ in the AI-based DSA group, with statistical difference between the two groups $(P<0.05)$. During the 3 -month follow-up period, in the conventional group, thrombosis occurred in 4 patients, low-flow AVF occurred in 5 patients, high-flow AVF occurred in 3 patients, and heart failure occurred in 5 patients. In the AI-based DSA group, thrombosis occurred in 2 patients, low-flow AVF occurred in 2 cases, high-flow AVF occurred in 1 case, and heart failure occurred in 3 cases. There were no other cardiac complications in both groups.

However, DSA requires high-dose X-ray radiation, which threatens the health of patients. DSA essentially refers to multiple frames of X-ray images, and DSA images contain many artifacts that affect doctors' diagnosis. Therefore, DSA must be performed again, which increases the radiation dose and reduces the doctor's diagnostic efficiency. How to obtain high-quality vascular images and reduce the radiation dose has become a hot spot $[19,20]$. The traditional method to remove DSA artifacts reduces vascular contrast. As a result, partial blood vessels are unclear. Artificial intelligence has found broad applications in the field of medical image processing and has a strong automatic learning function. In this study, in order to reduce the radiation doses during DSA and remove artifacts, the DSA images were optimized by an AI algorithm.

\section{Conclusion}

In this study, an AI algorithm was used to optimize DSA images, and its optimization effects were compared with the conventional method. It was found that the AI algorithm demonstrated better capabilities in removing the image artifacts, with low distortion rate and high resolution. The 
blood vessels were clearly displayed; in a word, it has guiding significance for the diagnosis and treatment of diseases.

\section{Data Availability}

The data used to support the findings of this study are available from the corresponding author upon request.

\section{Conflicts of Interest}

The author declares that there are no conflicts of interest.

\section{References}

[1] K. L. Ruedinger, S. Schafer, M. A. Speidel, and C. M. Strother, "4D-DSA: development and current neurovascular applications," American Journal of Neuroradiology, vol. 42, no. 2, pp. 214-220, 2021.

[2] M. Y. Yeung, "Pre-formed DSA and kidney allograft outcomes," Brazilian Journal of Nephrology, vol. 42, no. 2, pp. 136-137, 2020.

[3] J. F. Yu, L. Pung, H. Minami et al., "Virtual 2D angiography from four-dimensional digital subtraction angiography (4DDSA): a feasibility study," Interventional Neuroradiology, vol. 27, no. 2, pp. 307-313, 2021.

[4] X. Tian, B. Tian, Z. Shi et al., "Assessment of intracranial atherosclerotic plaques using 3D black-blood MRI: comparison with 3D time-of-F," Journal of Magnetic Resonance Imaging, vol. 53, no. 2, pp. 469-478, 2021.

[5] M. Vella, M. D. Alexander, M. C. Mabray et al., "Comparison of MRI, MRA, and DSA for detection of cerebral arteriovenous malformations in hereditary hemorrhagic telangiectasia," American Journal of Neuroradiology, vol. 41, no. 6, pp. 969-975, 2020.

[6] J. M. Yabu, "Should we use immunodominant DSA to measure response to therapy in antibody-mediated rejection?" Transplantation, vol. 104, no. 11, pp. 2260-2261, 2020.

[7] D. Song, C. Wu, L. Guo, L. Wang, J. Li, and X. Zhang, "Efficacy and safety of DSA-guided percutaneous sclerotherapy for venous malformations of penile region in children," Journal of Pediatric Surgery, vol. 56, no. 3, pp. 601-604, 2021.

[8] J. Zhang, S. Ding, H. Zhao et al., "Evaluation of chronic carotid artery occlusion by non-contrast 3D-MERGE MR vessel wall imaging: comparison with 3D-TOF-MRA, contrast-enhanced MRA, and DSA," European Radiology, vol. 30, no. 11, pp. 5805-5814, 2020.

[9] J. Qian, B. Song, Z. Jin, B. Wang, and H. Chen, "Linking empowering leadership to task performance, taking charge, and voice: the mediating role of feedback-seeking," Frontiers in Psychology, vol. 9, p. 2025, 2018.

[10] R. A. Lookstein, H. Haruguchi, K. Ouriel et al., "Drug-coated balloons for dysfunctional dialysis af," New England Journal of Medicine, vol. 383, no. 8, pp. 733-742, 2020.

[11] P. He, J.-p. Hu, H. Li et al., "Red blood cell distribution width and peritoneal dialysis-associated peritonitis prognosis," Renal Failure, vol. 42, no. 1, pp. 613-621, 2020.

[12] D. S. Hains, A. L. Schwaderer, A. E. Carroll et al., "Asymptomatic seroconversion of immunoglobulins to SARS-CoV-2 in a pediatric dialysis unit," Journal of the American Medical Association, vol. 323, no. 23, pp. 2424-2425, 2020.

[13] A. G. Reidenbach, M. F. Mesleh, D. Casalena et al., "Multimodal small-molecule screening for human prion protein binders," Journal of Biological Chemistry, vol. 295, no. 39, pp. 13516-13531, 2020.

[14] D. Jain, A. Rajab, J. S. Young et al., "Reversing donor-specific antibody responses and antibody-mediated rejection with bortezomib and belatacept in mice and kidney transplant recipients," American Journal of Transplantation, vol. 20, no. 10 , pp. $2675-2685,2020$.

[15] M. A. Wood-Trageser, Q. Xu, A. Zeevi, P. Randhawa, D. Lesniak, and A. J. Demetris, "Precision transplant pathology," Current Opinion in Organ Transplantation, vol. 25, no. 4, pp. 412-419, 2020.

[16] J. M. Zimmerer, M. W. Basinger, B. A. Ringwald et al., "Inverse abetween the quantity of human peripheral blood CXCR5+IFN- $\gamma+$ CD8+ T cells with de novo DSA production in the first year after kidney transplant," Transplantation, vol. 104, no. 11, pp. 2424-2434, 2020.

[17] K. Louis, C. Macedo, E. Bailly et al., "Coordinated circulating $\mathrm{T}$ follicular helper and activated $\mathrm{B}$ cell responses underlie the onset of antibody-mediated rejection in kidney transplantation," Journal of the American Society of Nephrology, vol. 31, no. 10, pp. 2457-2474, 2020.

[18] S. Lang, P. Hoelter, M. Schmidt et al., "Evaluation of an artificial intelligence-based 3D-angiography for visualization of cerebral vasculature," Clinical Neuroradiology, vol. 30, no. 4, pp. 705-712, 2020.

[19] J. H. Ng, V. Bijol, M. A. Sparks, M. E. Sise, H. Izzedine, and K. D. Jhaveri, "Pathophysiology and pathology of acute kidney injury in patients with COVID-19," Advances in Chronic Kidney Disease, vol. 27, no. 5, pp. 365-376, 2020.

[20] M. E. Haque, "Editorial for "assessment of intracranial atherosclerotic plaques using 3D black-blood MRI: comparison with 3D time-of-f", Journal of Magnetic Resonance Imaging, vol. 53, no. 2, pp. 479-480, 2021. 